\title{
Sum formulas involving powers of balancing and Lucas-balancing numbers - II
}

\author{
S. G. Rayaguru ${ }^{1}$ and G. K. Panda ${ }^{2}$ \\ ${ }^{1}$ Department of Mathematics, National Institute of Technology \\ Rourkela, India \\ e-mail: saigopalrs@gmail.com \\ ${ }^{2}$ Department of Mathematics, National Institute of Technology \\ Rourkela, India \\ e-mail: gkpanda_niterediffmail.com
}

Received: 15 November 2018

Revised: 6 May 2019

Accepted: 25 June 2019

\begin{abstract}
In this paper, we derive expressions for the sums of first four powers of balancing and Lucas-balancing numbers by using the telescoping summation formula. Further, we use these new results to obtain other closed form expressions studied earlier.
\end{abstract}

Keywords: Balancing numbers, Lucas-balancing numbers, Telescoping summation formula.

2010 Mathematics Subject Classification: 11B39.

\section{Introduction}

Balancing numbers were first introduced by Behera and Panda [3]. They called a natural number $n$, a balancing number if

$$
1+2+\cdots+(n-1)=(n+1)+(n+2)+\cdots+(n+r)
$$

holds for some natural number $r$. Further, if $x$ is a balancing number, then $\sqrt{8 x^{2}+1}$ is known as the Lucas-balancing number (see [9]). Moreover, $B_{n}$ and $C_{n}$ denote the $n$-th term of the balancing and Lucas-balancing sequence, respectively. For $n \geq 1$, the sequence of balancing and Lucas-balancing numbers satisfy the homogeneous linear recurrence $x_{n+1}=6 x_{n}-x_{n-1}$ with 
initial terms $B_{0}=0, B_{1}=1$ and $C_{0}=1, C_{1}=3$ respectively. For $\alpha=3+\sqrt{8}$ and $\beta=3-\sqrt{8}$, we have

$$
B_{n}=\frac{\alpha^{n}-\beta^{n}}{\alpha-\beta}, C_{n}=\frac{\alpha^{n}+\beta^{n}}{2},
$$

the closed form expression (or Binet form) of these sequences. Panda [9] proved many interesting properties of balancing and Lucas-balancing numbers, some of which resembles that of natural numbers and to some trigonometric identities.

Many authors studied the closed form expressions for different power sums involving Fibonacci and Lucas numbers (see [1,2,4,6-8]). In [5], Davala and Panda studied the sum and ratio formulas for balancing and Lucas-balancing numbers. Subsequently, Rayaguru and Panda [11] derived the closed form expressions for the power sums involving balancing and Lucas-balancing numbers. The authors also derived expressions for some infinite products involving these numbers (see [10]). In this paper, we obtain some new expressions for the non-alternating and alternating power sums of balancing and Lucas-balancing numbers.

First, we list some useful identities of balancing and Lucas-balancing numbers (see [11]).

Lemma 1.1. If $u$ and $v$ are integers, then
(1) $B_{-u}=-B_{u}$
(9) $B_{u+v} B_{u-v}=B_{u}^{2}-B_{v}^{2}$
(2) $C_{-u}=C_{u}$
(10) $B_{2 u}=2 B_{u} C_{u}$
(3) $C_{u}^{2}=8 B_{u}^{2}+1$
(11) $C_{2 u}=16 B_{u}^{2}+1$
(4) $C_{2 u}=2 C_{u}^{2}-1$
(12) $C_{3 u}=4 C_{u}^{3}-3 C_{u}$
(5) $B_{3 u}=32 B_{u}^{3}+3 B_{u}$
(13) $C_{u \pm v}=C_{u} C_{v} \pm 8 B_{u} B_{v}$
(6) $B_{u \pm v}=B_{u} C_{v} \pm C_{u} B_{v}$
(14) $B_{u+v}-B_{u-v}=2 C_{u} B_{v}$
(7) $B_{u+v}+B_{u-v}=2 B_{u} C_{v}$
(15) $C_{u+v}-C_{u-v}=16 B_{u} B_{v}$
(8) $C_{u+v}+C_{u-v}=2 C_{u} C_{v}$
(16) $8 B_{u+v} B_{u-v}=C_{u}^{2}-C_{v}^{2}$.

The following lemma deals with the telescoping summation identities required for obtaining the main results (see [6]).

Lemma 1.2. If $f(k)$ is a real sequence and $m$ and $n$ are positive integers, then

$$
\sum_{k=1}^{n}[f(m k+m)-f(m k-m)]=f(m n+m)+f(m n)-f(m)-f(0)
$$

and

$$
\sum_{k=1}^{n}(-1)^{k-1}[f(m k+m)-f(m k-m)]=(-1)^{n+1} f(m n+m)+(-1)^{n} f(m n)+f(m)-f(0) .
$$




\section{Sum formulas involving the powers of balancing and Lucas-balancing numbers}

In [11], the authors established different type of representations for the non-alternating and alternating summation formulas involving the powers of balancing and Lucas-balancing numbers. In this section, we explore other representations for the power sums of balancing and Lucas-balancing numbers. Further, we show that the results in [11] can be obtained from our new findings by using the properties of balancing and Lucas-balancing numbers.

Theorem 2.1. If $m$ and $n$ are positive integers, then
(a) $\sum_{k=1}^{n} B_{2 m k}=\frac{1}{B_{2 m}}\left[B_{m(n+1)}^{2}+B_{m n}^{2}\right]-\frac{B_{m}}{2 C_{m}}$
(b) $\sum_{k=1}^{n}(-1)^{k} B_{2 m k}=\frac{(-1)^{n}}{B_{2 m}}\left[B_{m(n+1)}^{2}-B_{m n}^{2}\right]-\frac{B_{m}}{2 C_{m}}$
(c) $\sum_{k=1}^{n} C_{2 m k}=\frac{1}{2 B_{2 m}}\left[B_{2 m(n+1)}+B_{2 m n}\right]-\frac{1}{2}$
(d) $\sum_{k=1}^{n}(-1)^{k} C_{2 m k}=\frac{(-1)^{n}}{2 B_{2 m}}\left[B_{2 m(n+1)}-B_{2 m n}\right]-\frac{1}{2}$.

Proof. Taking $f(k)=B_{k}^{2}$ in equation (1) of Lemma 1.2, we get

$$
\sum_{k=1}^{n}\left[B_{m k+m}^{2}-B_{m k-m}^{2}\right]=B_{m n+m}^{2}+B_{m n}^{2}-B_{m}^{2} .
$$

Applying Lemma 1.1 to the above summation, it follows that

$$
\sum_{k=1}^{n} B_{2 m k}=\frac{1}{B_{2 m}}\left[B_{m(n+1)}^{2}+B_{m n}^{2}-B_{m}^{2}\right]
$$

which completes the proof of (a). Now, taking $f(k)=B_{2 k+2 m}$ in equation (1) of Lemma 1.2 and proceeding as above, it is easy to show that

$$
\begin{aligned}
& 2 B_{2 m} \sum_{k=1}^{n} C_{2 m(k+1)}=B_{2 m(n+2)}+B_{2 m(n+1)}-B_{4 m}-B_{2 m} \\
\Longrightarrow & 2 B_{2 m}\left[\sum_{k=1}^{n+1} C_{2 m k}-C_{2 m}\right]=B_{2 m(n+2)}+B_{2 m(n+1)}-B_{4 m}-B_{2 m} \\
\Longrightarrow & 2 B_{2 m} \sum_{k=1}^{n+1} C_{2 m k}=B_{2 m(n+2)}+B_{2 m(n+1)}-B_{2 m},
\end{aligned}
$$

which gives (c) after replacing $(n+1)$ by $n$. The proofs of (b) and (d) are similar to the proofs of (a) and (c), respectively, using equation (2) of Lemma 1.2. Hence, we omit the proof. 
From the proof of the above theorem, it can be seen that

$$
\sum_{k=1}^{n} B_{2 m k}=\frac{B_{m n+m}^{2}+B_{m n}^{2}-B_{m}^{2}}{B_{2 m}} .
$$

Using the properties of balancing and Lucas-balancing numbers from Lemma 1.1, it is easy to show that

$$
\begin{aligned}
\sum_{k=1}^{n} B_{2 m k} & =\frac{B_{m n+m}\left(B_{m n+m}+B_{m n-m}\right)}{B_{2 m}} \\
& =\frac{B_{m n+m} \cdot 2 B_{m n} C_{m}}{B_{2 m}}=\frac{B_{m n+m} B_{m n}}{B_{m}} .
\end{aligned}
$$

Similarly,

$$
\begin{aligned}
\sum_{k=1}^{n} C_{2 m k} & =\frac{B_{2 m n+2 m}+B_{2 m n}-B_{2 m}}{2 B_{2 m}} \\
& =\frac{B_{2 m n}+2 B_{m n} C_{m n+2 m}}{2 B_{2 m}} \\
& =\frac{B_{m n}\left(C_{m n}+C_{m n+2 m}\right)}{B_{2 m}} \\
& =\frac{B_{m n} \cdot 2 C_{m} C_{m n+m}}{B_{2 m}}=\frac{C_{m n+m} B_{m n}}{B_{m}} .
\end{aligned}
$$

The above two summation results appear in [11, Theorem 3.1]. The corresponding alternating versions can be modified in a similar fashion to obtain the results in [11, Theorem 3.2].

The following is an immediate consequence of Theorem 2.1 .

Corollary 2.1.1. If $m$ and $n$ are positive integers, then

(a) $6 \sum_{k=1}^{n} B_{2 k}=B_{n+1}^{2}+B_{n}^{2}-1$

(b) $6 \sum_{k=1}^{n}(-1)^{k} B_{2 k}=(-1)^{n}\left(B_{n+1}^{2}-B_{n}^{2}\right)-1$,

(c) $12 \sum_{k=1}^{n} C_{2 k}=B_{2 n+2}+B_{2 n}-6$,

(d) $12 \sum_{k=1}^{n}(-1)^{k} C_{2 k}=(-1)^{n}\left(B_{2 n+2}-B_{2 n}\right)-6$.

The following theorem deals with the sums of balancing and Lucas-balancing squares.

Theorem 2.2. If $m$ and $n$ are positive integers, then

(a) $\sum_{k=1}^{n} B_{m k}^{2}=\frac{1}{32 B_{2 m}}\left[B_{2 m(n+1)}+B_{2 m n}\right]-\frac{1+2 n}{32}$, 
(b) $\sum_{k=1}^{n}(-1)^{k} B_{m k}^{2}=\frac{(-1)^{n}}{32 B_{2 m}}\left[B_{2 m(n+1)}-B_{2 m n}\right]+\frac{(-1)^{n+1}}{32}$,

(c) $\sum_{k=1}^{n} C_{m k}^{2}=\frac{1}{4 B_{2 m}}\left[B_{2 m(n+1)}+B_{2 m n}\right]+\frac{-1+2 n}{4}$,

(d) $\sum_{k=1}^{n}(-1)^{k} C_{m k}^{2}=\frac{(-1)^{n}}{4 B_{2 m}}\left[B_{2 m(n+1)}-B_{2 m n}\right]+\frac{-2+(-1)^{n}}{4}$.

Proof. Using the identity $16 B_{u}^{2}=C_{2 u}-1$, it follows that

$$
16 \sum_{k=1}^{n} B_{m k}^{2}=\sum_{k=1}^{n} C_{2 m k}-n \text {. }
$$

Applying Theorem 2.1 to the above summation, the identity in (a) follows immediately. The proof of (c) is similar to the proof of (a), by considering the alternating summation

$$
16 \sum_{k=1}^{n}(-1)^{k-1} B_{m k}^{2}=\sum_{k=1}^{n}(-1)^{k-1} C_{2 m k}-\frac{(-1)^{n-1}+1}{2} .
$$

The proofs of (b) and (d) are similar to those of (a) and (c), respectively, from the identity $2 C_{u}^{2}=C_{2 u}+1$. Hence, we omit their proofs.

From the above theorem, it can be seen that

$$
\begin{aligned}
16 \sum_{k=1}^{n} B_{m k}^{2} & =\frac{B_{2 m n+2 m}+B_{2 m n}-B_{2 m}}{2 B_{2 m}}-n \\
& =\frac{C_{m n+m} B_{m n}}{B_{m}}-n
\end{aligned}
$$

which appears in [11, Theorem 3.5]. The corresponding summation identities for Lucas-balancing numbers and the alternating versions can be modified in a similar manner to obtain other results in [11, Theorem 3.5].

The following is an immediate consequence of Theorem 2.2.

Corollary 2.2.1. If $m$ and $n$ are positive integers, then

(a) $192 \sum_{k=1}^{n} B_{k}^{2}=B_{2 n+2}+B_{2 n}-6(2 n+1)$,

(b) $192 \sum_{k=1}^{n}(-1)^{k} B_{k}^{2}=(-1)^{n}\left(B_{2 n+2}-B_{2 n}\right)-6(-1)^{n}$,

(c) $24 \sum_{k=1}^{n} C_{k}^{2}=B_{2 n+2}+B_{2 n}+6(2 n-1)$,

(d) $24 \sum_{k=1}^{n}(-1)^{k} C_{k}^{2}=(-1)^{n}\left(B_{2 n+2}-B_{2 n}\right)+6\left((-1)^{n}-2\right)$. 
The following theorem deals with the sums of balancing and Lucas-balancing cubes.

Theorem 2.3. If $m$ and $n$ are positive integers, then

(a) $\sum_{k=1}^{n} B_{2 m k}^{3}=\frac{1}{32 B_{6 m}}\left[B_{3 m(n+1)}^{2}+B_{3 m n}^{2}\right]-\frac{3}{32 B_{2 m}}\left[B_{m(n+1)}^{2}+B_{m n}^{2}\right]-\frac{B_{3 m}}{64 C_{3 m}}+\frac{3 B_{m}}{64 C_{m}}$,

(b) $\sum_{k=1}^{n}(-1)^{k} B_{2 m k}^{3}=\frac{(-1)^{n}}{32 B_{6 m}}\left[B_{3 m(n+1)}^{2}-B_{3 m n}^{2}\right]-\frac{3(-1)^{n}}{32 B_{2 m}}\left[B_{m(n+1)}^{2}-B_{m n}^{2}\right]-\frac{B_{3 m}}{64 C_{3 m}}+\frac{3 B_{m}}{64 C_{m}}$,

(c) $\sum_{k=1}^{n} C_{2 m k}^{3}=\frac{1}{8 B_{6 m}}\left[B_{6 m(n+1)}+B_{6 m n}\right]+\frac{3}{8 B_{2 m}}\left[B_{2 m(n+1)}+B_{2 m n}\right]-\frac{1}{2}$,

(d) $\sum_{k=1}^{n}(-1)^{k} C_{2 m k}^{3}=\frac{(-1)^{n}}{8 B_{6 m}}\left[B_{6 m(n+1)}-B_{6 m n}\right]+\frac{3(-1)^{n}}{8 B_{2 m}}\left[B_{2 m(n+1)}-B_{2 m n}\right]-\frac{1}{2}$.

Proof. Using the identities $32 B_{u}^{3}=B_{3 u}-3 B_{u}$ and $4 C_{u}^{3}=C_{3 u}+3 C_{u}$, respectively, proof of this theorem follows from the results obtained in Theorem 2.1 and the proof of Theorem 2.2. Hence, we omit the proof.

From the above theorem, it can be seen that

$$
\begin{aligned}
\sum_{k=1}^{n} B_{2 m k}^{3} & =\frac{B_{3 m n+3 m}^{2}+B_{3 m n}^{2}-B_{3 m}^{2}}{32 B_{6 m}}-\frac{3\left(B_{m n+m}^{2}+B_{m n}^{2}-B_{m}^{2}\right)}{32 B_{2 m}} \\
& =\frac{B_{3 m n} B_{3 m n+3 m}}{32 B_{3 m}}-\frac{3 B_{m n} B_{m n+m}}{32 B_{m}} .
\end{aligned}
$$

Using the identity $B_{3 u}=B_{u}\left(32 B_{u}^{2}+3\right)$, we have

$$
B_{3 m n} B_{3 m n+3 m}=B_{m n} B_{m n+m}\left[\left(32 B_{m n} B_{m n+m}\right)^{2}+96\left(B_{m n}^{2}+B_{m n+m}^{2}\right)+9\right]
$$

and hence

$$
\begin{aligned}
\sum_{k=1}^{n} B_{2 m k}^{3} & =\frac{B_{m n} B_{m n+m}}{32 B_{3 m}}\left[\left(32 B_{m n} B_{m n+m}\right)^{2}+96\left(B_{m n}^{2}+B_{m n+m}^{2}-B_{m}^{2}\right)\right] \\
& =\frac{B_{m n} B_{m n+m}}{B_{3 m}}\left[2 B_{m n} B_{m n+m}\left(16 B_{m n} B_{m n+m}+3 C_{m}\right)\right] \\
& =\frac{2 B_{m n}^{2} B_{m n+m}^{2}}{B_{3 m}}\left[16 B_{m n} B_{m n+m}+3 C_{m}\right] \\
& =\frac{2 B_{m n}^{2} B_{m n+m}^{2}}{B_{3 m}}\left[C_{2 m n+m}+2 C_{m}\right] \\
& =\frac{2 B_{m n}^{2} B_{m n+m}^{2}}{B_{3 m}}\left[2 C_{m n} C_{2 m n+m}+C_{m}\right]
\end{aligned}
$$


which appears in [11, Theorem 3.9]. Further,

$$
\begin{aligned}
\sum_{k=1}^{n} B_{2 m k}^{3} & =\frac{2 B_{m n}^{2} B_{m n+m}^{2}}{B_{3 m}}\left[2 C_{m n} C_{2 m n+m}+C_{m}\right] \\
& =\frac{B_{m n} B_{m n+m}}{4 B_{3 m}}\left[16 B_{m n} B_{m n+m} C_{m n} C_{m n+m}+8 C_{m} B_{m n} B_{m n+m}\right] \\
& =\frac{2 B_{m n}^{2} B_{m n+m}^{2}}{B_{3 m}}\left[4 B_{2 m n} B_{2 m n+2 m}+8 C_{m} B_{m n} B_{m n+m}\right] \\
& =\frac{2 B_{m n}^{2} B_{m n+m}^{2}}{B_{3 m}}\left[\frac{C_{m}\left(C_{2 m n+m}-C_{m}\right)}{2}+\frac{C_{4 m n+2 m}-C_{2 m}}{4}\right] \\
& =\frac{2 B_{m n}^{2} B_{m n+m}^{2}}{B_{3 m}}\left[\frac{C_{m} C_{2 m n+m}-C_{m}^{2}}{2}+\frac{C_{2 m n+m}^{2}-C_{m}^{2}}{2}\right] \\
& =\frac{2 B_{m n}^{2} B_{m n+m}^{2}}{B_{3 m}}\left[C_{m n} C_{m n+m} C_{2 m n+m}-C_{m}^{2}\right]
\end{aligned}
$$

which appears in [11, Theorem 3.7]. Proceeding as above, corresponding summation formulas for Lucas-balancing numbers and the alternating versions can be obtained, which appears in [11, Theorem 3.7, Theorem 3.9, Theorem 3.13, Theorem 3.15].

An immediate consequence of Theorem 2.3 is as follows.

Corollary 2.3.1. If $m$ and $n$ are positive integers, then

(a) $221760 \sum_{k=1}^{n} B_{2 k}^{3}=\left[B_{3 n+3}^{2}+B_{3 n}^{2}-3\left(B_{n+1}^{2}+B_{n}^{2}\right)\right]+2240$,

(b) $221760 \sum_{k=1}^{n}(-1)^{k} B_{2 k}^{3}=(-1)^{n}\left[B_{3 n+3}^{2}-B_{3 n}^{2}-3\left(B_{n+1}^{2}-B_{n}^{2}\right)\right]+2240$,

(c) $55440 \sum_{k=1}^{n} C_{2 k}^{3}=\left[B_{6 n+6}+B_{6 n}+3\left(B_{2 n+2}+B_{2 n}\right)\right]-27720$,

(d) $55440 \sum_{k=1}^{n}(-1)^{k} C_{2 k}^{3}=(-1)^{n}\left[B_{6 n+6}-B_{6 n}+3\left(B_{2 n+2}-B_{2 n}\right)\right]-27720$.

In the following theorem, we explore the non-alternating and alternating summations involving the fourth power of balancing and Lucas-balancing numbers.

Theorem 2.4. If $m$ and $n$ are positive integers, then

(a) $\sum_{k=1}^{n} B_{m k}^{4}=\frac{1}{1024 B_{4 m}}\left[B_{4 m(n+1)}+B_{4 m n}\right]-\frac{1}{256 B_{2 m}}\left[B_{2 m(n+1)}+B_{2 m n}\right]+\frac{6 n+3}{1024}$,

(b) $\sum_{k=1}^{n}(-1)^{k} B_{m k}^{4}=\frac{(-1)^{n}}{1024 B_{4 m}}\left[B_{4 m(n+1)}-B_{4 m n}\right]-\frac{(-1)^{n}}{256 B_{2 m}}\left[B_{2 m(n+1)}-B_{2 m n}\right]+\frac{3(-1)^{n}}{1024}$,

(c) $\sum_{k=1}^{n} C_{m k}^{4}=\frac{1}{B_{4 m}}\left[B_{4 m(n+1)}+B_{4 m n}\right]+\frac{1}{B_{2 m}}\left[B_{2 m(n+1)}+B_{2 m n}\right]+\frac{6 n-5}{1024}$, 
(d) $\sum_{k=1}^{n}(-1)^{k} C_{m k}^{4}=\frac{(-1)^{n}}{B_{4 m}}\left[B_{4 m(n+1)}-B_{4 m n}\right]+\frac{(-1)^{n}}{B_{2 m}}\left[B_{2 m(n+1)}-B_{2 m n}\right]+\frac{3(-1)^{n}}{1024}-\frac{1}{128}$.

Proof. In view of Lemma 1.1, we have

$$
16 B_{u}^{2}=C_{2 u}-1 \quad \text { and } \quad 2 C_{u}^{2}=C_{2 u}+1
$$

Upon squaring these identities, we get

$$
256 B_{u}^{4}=C_{2 u}^{2}-2 C_{2 u}+1 \quad \text { and } \quad 4 C_{u}^{4}=C_{2 u}^{2}+2 C_{2 u}+1
$$

respectively. Using the results obtained in Theorem 2.1, proof of this theorem is similar to the proof of Theorem 2.3 and hence, we omit the proof.

From the above theorem, it can be seen that

$$
\begin{aligned}
512 \sum_{k=1}^{n} B_{m k}^{4} & =\frac{B_{4 m n+4 m}+B_{4 m n}}{2 B_{4 m}}-\frac{2\left(B_{2 m n+2 m}+B_{2 m n}\right)}{B_{2 m}}+\frac{6 n+3}{2} \\
& =\frac{B_{4 m n+2 m} C_{2 m}}{B_{4 m}}-\frac{4 B_{2 m n+m} C_{m}}{B_{2 m}}+\frac{6 n+3}{2} \\
& =\frac{B_{2 m n+m}\left(C_{2 m n+m}-4 C_{m}\right)}{B_{2 m}}+\frac{6 n+3}{2}
\end{aligned}
$$

which appears in [11, Theorem 3.19]. In a similar fashion, corresponding summation for Lucasbalancing numbers and the alternating versions can be obtained, which appears in [11, Theorem 3.19, Theorem 3.21, Theorem 3.22].

The following is an immediate consequence of Theorem 2.4 .

Corollary 2.4.1. If $m$ and $n$ are positive integers, then
(a) $208896 \sum_{k=1}^{n} B_{k}^{4}=\left[B_{4 n+4}+B_{4 n}-136\left(B_{2 n+2}+B_{2 n}\right)+612(2 n+1)\right]$,
(b) $208896 \sum_{k=1}^{n}(-1)^{k} B_{k}^{4}=(-1)^{n}\left[B_{4 n+4}-B_{4 n}-136\left(B_{2 n+2}-B_{2 n}\right)+612\right]$,
(c) $52224 \sum_{k=1}^{n} C_{k}^{4}=\left[256\left(B_{4 n+4}+B_{4 n}\right)+8704\left(B_{2 n+2}+B_{2 n}\right)+51(6 n-5)\right]$,
(d) $52224 \sum_{k=1}^{n}(-1)^{k} C_{k}^{4}=(-1)^{n}\left[256\left(B_{4 n+4}-B_{4 n}\right)+8704\left(B_{2 n+2}-B_{2 n}\right)+153\right]-408$.

\section{Acknowledgements}

The authors are very much thankful to the anonymous referees and editor for their valuable suggestions which improved the presentation of the paper. 


\section{References}

[1] Adegoke, K. (2018). Factored closed-form expressions for the sums of cubes of Fibonacci and Lucas numbers, J. Int. Seq., 21, Article 18.6.8.

[2] Adegoke, K. (2017). Sums of fourth powers of Fibonacci and Lucas numbers (Preprint), Available online at: https://arxiv.org/pdf/1706.00407.pdf.

[3] Behera, A., \& Panda, G. K. (1999). On the square roots of triangular numbers, Fib. Quart., 37 (2), 98-105.

[4] Clary, S., \& Hemenway, P. D. (1993). On sums of cubes of Fibonacci numbers, in Applications of Fibonacci Numbers, Kluwer Academic Publishers, Dordrecht, The Netherlands, 5, 123-136.

[5] Davala, R. K., \& Panda, G. K. (2015). On sum and ratio formulas for balancing numbers, Journal of the Ind. Math. Soc., 82 (1-2), 23-32.

[6] Frontczak, R. (2018). Sums of powers of Fibonacci and Lucas numbers: A new bottom-up approach, Notes Number Theory Discrete Math., 24 (2), 94-103.

[7] Frontczak, R. (2018). Sums of cubes over odd-index Fibonacci numbers, Integers, 18, \#A36.

[8] Melham, R.S. (2000). Alternating sums of fourth powers of Fibonacci and Lucas numbers, Fib. Quart., 38 (3), 254-259.

[9] Panda, G. K. (2009). Some fascinating properties of balancing numbers, Proc. of Eleventh Internat. Conference on Fibonacci Numbers and Their Applications, Cong. Numerantium, 194, 185-189.

[10] Rayaguru, S. G., \& Panda, G. K. (2018). Some infinite product identities involving balancing and Lucas-balancing numbers, Alabama Journal of Mathematics, 42, 6 pages.

[11] Rayaguru, S. G., \& Panda, G. K. (2019). Sum formulas involving powers of balancing and Lucas-balancing numbers, Journal of the Ind. Math. Soc., 86 (1-2), 137-160. 